Case Report

\title{
Diagnostic challenge of follicular thyroid carcinoma with insular component mixed with follicular patterned papillary carcinoma
}

\author{
Sadek S.A. ${ }^{1}$, Haider N. ${ }^{2}$, Fatima S. ${ }^{3}$ \\ ${ }^{1}$ Dr. Shaymaa Ahmed Sadek, Aseer Central Hospital, Abha, KSA, ${ }^{2}$ Dr. Nazima Haider, King Khalid University, Abha, \\ KSA, ${ }^{3}$ Dr. Sohaila Fatima, King Khalid University, Abha, KSA.
}

Correspondence Author: Dr. Nazima Haider, Aseer Central Hospital, King Khalid University, Abha, KSA. Email: nazima_haider@yahoo.com

\begin{abstract}
Thyroid tumours with mixed follicular histological components present a great challenge for pathologists. Poorly differentiated carcinoma (or insular carcinoma) is intermediate tumour between well differentiated and anaplastic carcinomas of thyroid which may be associated with papillary and follicular thyroid carcinoma. It is important to differentiate the insular component and medullary carcinoma. We present an uncommon case of mixed follicular thyroid carcinoma with an insular component and follicular papillary variant in a 30 year old female which cannot be accurately diagnosed without application of immunohistochemistry.
\end{abstract}

Keywords: Mixed follicular thyroid carcinoma, Insular component, Follicular papillary

\section{Introduction}

Accurate classification of follicular thyroid lesions is a diagnostic challenge for the pathologists especially in resource challenged environments. It is not always an easy task on routine hematoxylin and eosin (H\&E) specimens. Although the differences and diagnostic criteria are clearly mentioned in books but many findings are subjective and differential diagnosis criteria subtle. Interobservor or intraobservor disagreements in the diagnosis of follicular thyroid lesions are common, even among expert pathologists [1]. Thyroid follicular cellmalignant tumours are classified as well- differentiated (papillary and follicular carcinoma) or undifferentiated (anaplastic). Poorly differentiated carcinoma (or insular carcinoma) exists as a group of in-between these two types [2]. We present an uncommon case of mixed follicular thyroid carcinoma with an insular component and follicular papillary variant in a 30 year old female and discuss the diagnostic challenges for a pathologist in diagnosing such tumours, which cannot be accurately diagnosed without application of immunohistochemistry.

\section{Case Report}

A 36 year old female with left sided nodular, firm thyroid mass slowly increasing from 2 years was diagnosed with follicular neoplasm on Fine needle aspiration cytology (FNAC). The mass was removed by total thyroidectomy. Thyroid measuring $12.4 \times 7.7 \times 4.5 \mathrm{~cm}$ was received in histopathology lab. Gross examination showed a firm rounded nodular thyroid mass measuring $8 \mathrm{~cm} \times 6 \mathrm{~cm} \times 4 \mathrm{~cm}$ in the thyroid. Cut section showed right lobe and isthmus showed vaguely nodular pattern with colloid. Left lobe showed tan colours soft mass, $5 \times 4 \times 2 \mathrm{~cm}$, nearly occupying whole of left lobe leaving a thin rim of normal thyroid tissue. Focal interruption of capsule was seen grossly. Microscopic sections of the right lobe showed two microscopic foci of follicular type of papillary carcinoma with characteristic nuclear features and remaining tissue was multiple colloid filled nodules with focal hyperplastic change and chronic inflammation. Left sided nodular mass showed follicular neoplasm with capsule invasion at places by tongues of tumour cells. There were areas composed of solid large nests (insulae) comprised of relatively monotonous small cells with round nuclei, faint chromatin, small nucleoli and eosinophilic cytoplasm (Fig 1B \& 2B) along with follicular and microfollicular areas (Figure 1A \& 1B). Occasional cells showed nuclear groove. There was no necrosis and mitosis is occasional. Few areas of follicular variant of papillary carcinoma were also seen. (3A, 3B, 3D) In some areas, the lobules were separated by

Manuscript received: $10^{\text {th }}$ July 2018

Reviewed: $20^{\text {th }}$ July 2018

Author Corrected: $26^{\text {th }}$ July 2018

Accepted for Publication: $30^{\text {th }}$ July 2018

Pathology Update: Tropical Journal of Pathology \& Microbiology Available online at: www.pathologyreview.in 427 | P a g e 


\section{Case Report}

eosinophilic amorphous substance resembling amyloid. (Fig 2A). The relative proportion of solid insular pattern was around $50 \%$. Areas of capsular invasion were showing insular pattern. However, there was no lympho-vascular invasion. There was no evidence of local invasion or metastasis either. Her serum levels of thyroglobulin antigen were $15.5 \mathrm{ng} / \mathrm{mL}$ (normal range, 1.4 to 78.0 ) and his serum calcitonin level was $4.2 \mathrm{pg} / \mathrm{mL}$ (normal range, $<10$ ).

No definite diagnosis was made as none of the tumour criteria was fully satisfied. Immunohistochemistry showed whole of the tumour to be thyroglobulin positive with weak positivity in solid insular areas. (Fig 1C \& 1D) Neuron specific Enolase (NSE) (Fig 2D) was positive in insular areas.

Foci of papillary carcinoma were more strongly CK19 positive (Fig 3C) All other neuroendocrine markers- calcitonin, (Fig 2 C) synaptophysin, chromogranin and S100 were negative. A diagnosis of minimally invasive follicular carcinoma with insular pattern with foci of microscopicfollicular variant of papillary carcinoma was made.

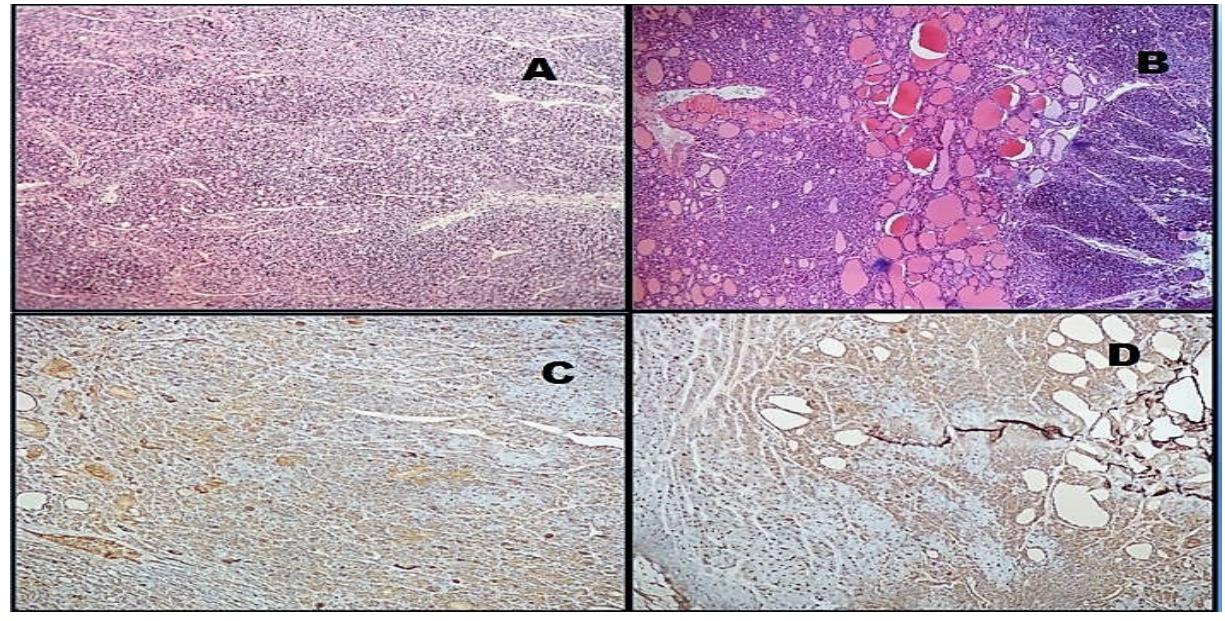

Figure 1A: Microfollicular patterns. (Hematoxylin and Eosin (H\&E), 10X)

Figure 1B: Mixed solid areas of large nests (insulae) comprised of relatively monotonous small cells [right side] with follicular pattern. [left side]. (H\&E, 20X)

Figure 1C, D: Thyroglobulin positivity with weak positivity in solid insular areas. (Immunohistochemistry, 10X)

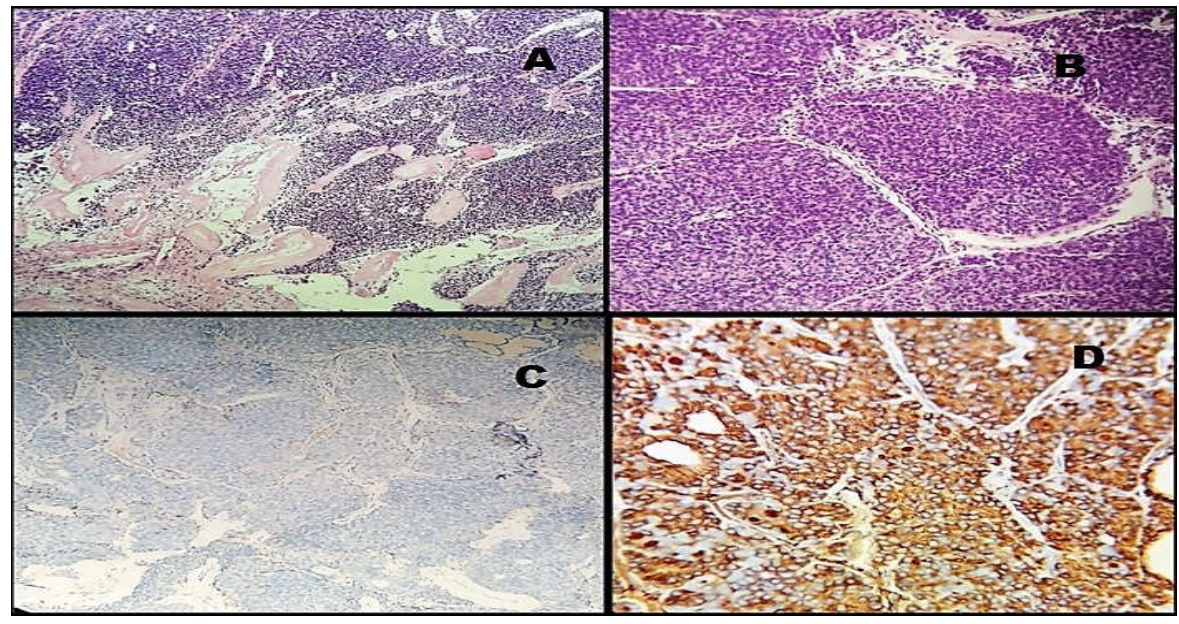

Figure 2A: Areas of lobules separated by eosinophilic amorphous substance resembling amyloid. (H\&E, 10X)

Figure 2B: Large nests (insulae) comprised of relatively monotonous small cells. (H\&E,20X)

Figure 2C: Cells forming nests are calcitonin negative. (Immunohistochemistry, 10X)

Figure 2D: Cells forming nests are NSE positive. (immunohistochemistry, 20X) 


\section{Case Report}

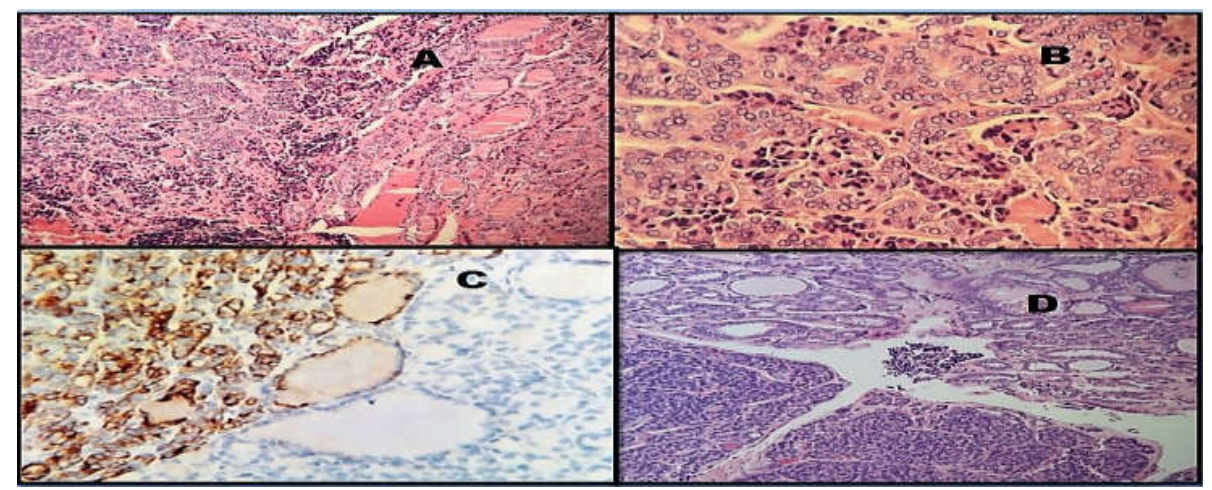

Figure 3A, B: Areas of follicular variant of papillary carcinoma with clear nuclei, nuclear grooves. (H\&E, 10X \& 20X) Figure 3C: Areas of follicular variant of papillary carcinoma are positive for CK 19. (Immunohistochemistry, 10X) Figure 3D: Areas of follicular variant of papillary carcinoma with insular component. (H\&E, 10X)

\section{Discussion}

Histopathological analysis of follicular patterns of thyroid tumors is difficult. Studies have shown considerable interobserver and intraobserver variability as many differentiating feature are subtle and subjective [1]. Poorly differentiated carcinoma (or insular carcinoma) is intermediate tumour between well differentiated and anaplastic carcinomas of thyroid. Insular growth pattern may be associated with papillary and follicular thyroid carcinoma [2]. However, thyroid tumours with these mixed follicular histological components present a great challenge for pathologists [1]. One of the rare challenging tumour is mixed medullary-follicular-papillary carcinoma. Although it's very rare, our case resembled it a lot on histopathological examination [3]. It is very important to differentiate the insular component and medullary carcinoma as the treatment and prognosis are completely different and presence of insular pattern with follicular carcinoma carries a poor prognosis. The diagnosis can only be confirmed by immunohistochemistry and molecular studies $[1,3]$.

The presence of an insular component together with typical follicular carcinoma could either be a coincident finding of a mixed tumour or a follicular thyroid carcinoma progressing into a poorly differentiated form $[1,3]$.

Insular carcinoma is rare with $0.3-6.7 \%$ of all thyroid carcinomas and is seen in older patients, mean age 5563 years. Diagnostic criteria for Insular or poorly differentiated carcinoma are (a) presence of a solid/ trabecular/insular pattern of growth, (b) absence of the conventional nuclear features of papillary carcinoma, and (c) presence of at least one of the following features: convoluted nuclei; mitotic activity $>$ or $=3$ per $10 \mathrm{HPF}$; and tumor necrosis [4]. Cells are positive for CK 7, Thyroglobulin (faint), TTF 1, NSE, Ki67 - 10-
$30 \%$ with negativity for calcitonin and parathyroid hormone [5]. In our case except for the insular growth pattern, other features were not present. Nuclei were round with faint to pale chromatin, mitosis was low and no necrosis was seen and cells were faint thyroglobulin, NSE positive and synaptophysin, chromogranin, S100, calcitonin negative.

PAX8-PPAR and RET/PTC gene rearrangements are recognized to represent initiating events in the carcinogeneses of thyroid follicular carcinoma and papillary carcinoma, respectively. Insular carcinoma does not show evidence of RET/PTC fusion gene rearrangement thus suggesting that well-differentiated thyroid carcinomas associated with these gene arrangements do not progress to the dedifferentiated form $[6,7]$. However, some studies have shown RET/PTC expression especially with histologic evidence of coexistent papillary carcinoma or only with the cardinal nuclear features of papillary carcinoma, which implies a possible evolution from papillary carcinoma and suggestion that it is related to both follicular and papillary carcinoma, not exclusively limited to follicularcarcinoma [8].

Important differential diagnoses of insular pattern are anaplastic carcinoma, medullary carcinoma, Hurthle cell tumours, parathyroid carcinoma, solid variant of papillary carcinoma. It is challenging to differentiate it from anaplastic thyroid carcinomas which have highly pleomorphic nuclear features, lack of follicular differentiation and are thyroglobulin and TTF-1 negative [9].

Medullary carcinoma which is another close differential has prominent vasculature, granular cytoplasm, stippled chromatin and calcitonin, synaptophysin, chromogranin A and amyloid positive and thyroglobulin negative [5]. 


\section{Case Report}

Synaptophysin, chromogranin A, CK 7, CK 20, CD56, NSE, carcinoembryonic antigen (CEA), S100, RET, PPAR, calcitonin, galectin-3, and thyroglobulin are the important immune markers for differentiating thyroid carcinomas. Insular or poorly differentiated carcinomas are CK 7, thyroglobulin, NSE positive in most cases while other markers are usually negative. Calcitonin and S100 may be weakly positive in some cases and one needs knowledge of variable immunohistochemical expressions and clinicopathologic correlations to confirm the diagnosis. $[5,7]$.

There is no individual biomarker having sufficient sensitivity or specificity to distinguish benign from malignant lesions or different types of malignancy. The application of a panel of immunomarkers improves the differential power of individual markers and aids in the accurate classification of challenging thyroid lesions [10].

\section{Conclusion}

- Accurate classification of follicular thyroid lesions is a diagnostic challenge for the pathologists.

- Insular growth pattern may be associated with papillary and follicular thyroid carcinoma.

- The presence of an insular component together with typical follicular carcinoma could either be a coincident finding of a mixed tumour or a follicular thyroid carcinoma progressing into a poorly differentiated form.

- It is very important to differentiate the insular component and medullary carcinoma as the treatment and prognosis are completely different.

Funding: Nil, Conflict of interest: None initiated Permission from IRB: Yes

\section{References}

1. Ishikawa Y. Ciliated muconodular papillary tumor of the peripheral lung: benign or malignant. Pathol. Clin. Med. (Byouri- to - Rinsho) 2002; 20: 964-965. (In Japanese).
2. Ishikawa M, Sumitomo S, Imamura N, Nishida T, Mineura K, Ono K. Ciliated muconodular papillary tumor of the lung: report of five cases. Journal of Surgical Case Reports 2016;8:1-4. doi: 10.1093/jscr/ rjw144.

3. Flieder DB, Thivolet-Bejui F, Popper H. Tumor of the lung. Benign epithelial lesions. In: Travis WD, Brambilla E, Müller-Hermelink HK, Harris CC, editors. World Health Organization classification of tumors: pathology and genetics of tumors of the lung, pleura, thymus and heart. Lyon: IARC Press 2004: 78-81.

4. Nakamura S, Koshikawa T, Sato T, Hayashi K, Suchi T. Extremely well differentiated papillary adenocarcinoma of the lung with prominent cilia formation. Acta PatholJpn. 1992 Oct;42(10):745-50.

5. Kamata T, Yoshida A, Kosuge T, Watanabe S, Asamura H, Tsuta K. Ciliated muconodular papillary tumors of the lung: a clinicopathologic analysis of 10 cases. Am J SurgPathol. 2015 Jun; 39(6):753-60. doi: 10.1097/PAS.0000000000000414.

6. Lau KW, Aubry MC, Tan GS, Lim CH, Takano AM. Ciliated muconodular papillary tumor: a solitary peripheral lung nodule in a teenage girl.Hum Pathol. 2016 Mar;49:22-6.doi: 10.1016/j.humpath.2015.09.038. Epub 2015 Oct 28.

7. Sato T, Koike K, Homma A, Yokoyama A. Ciliated muconodular papillary tumour of the lung: a newly defined low-grade malignant tumour. Interact Cardiovasc Thorac Surg 2010; 11 (5): 685-687.doi: 10. 1510 / icvts. 2009.229989.

8. Arai Y, Shimizu S, Eimoto T, Shimizu S, Otsuki Y, Kobayashi H, Ogawa H, Travis WD. Peripheral pulmonary papillary/glandular neoplasms with ciliated cells and a component of well-differentiated adenocarcinoma: report of three tumours. Histopathology. 2010 Jan; 56 (2): 265-9. doi: 10. 1111/j. 1365-2559.2009.03463.x.

\section{How to cite this article?}

Sadek S.A, Haider N, Fatima S. Diagnostic challenge of follicular thyroid carcinoma with insular component mixed with follicular patterned papillary carcinoma. Trop J Path Micro 2018; 4(6):427-430.doi:10.17511/jopm.2018.i06.01 